\title{
Polarity imbalance of the photospheric magnetic field
}

\author{
ElenaVernova ${ }^{1} \cdot$ Marta Tyasto $^{1}$. \\ Dmitrii Baranov ${ }^{2}$. Olga Danilova ${ }^{1}$
}

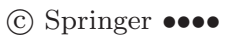

\begin{abstract}
Polarity imbalance of the photospheric magnetic field was studied using synoptic maps of NSO Kitt Peak (1976 - 2016). Imbalance of positive and negative fluxes was considered for the fields with strength $B>50 \mathrm{G}$ in the sunspot zone $\left(5^{\circ}-40^{\circ}\right)$ and for the fields with strength $B<50 \mathrm{G}$ at higher latitudes $\left(40^{\circ}-90^{\circ}\right)$. The 22-year periodicity in the imbalance of positive and negative fields was found which maintained itself during four solar cycles. While for the sunspot zone the sign of the imbalance always coincides with the northern hemisphere polarity, for the high latitudes the sign of the imbalance always coincides with the southern hemisphere polarity. Good correspondence of the flux imbalance with the quadrupole moment $\left(g_{20}\right)$ of the potential-field sourcesurface (PFSS) model was observed. The polarity imbalance of the sunspot zone correlates, on one hand, with the asymmetry of the magnetic field of the Sunas-a-star and, on the other hand, with the sector structure of the interplanetary magnetic field. The obtained results show the close connection of the magnetic fields in active regions with the Sun's polar magnetic field. The weakest fields $B<5 \mathrm{G}$ represent quite a special group with the magnetic flux developing in antiphase to the fluxes of the stronger fields.
\end{abstract}

Keywords: Magnetic fields, Photosphere; Polarity imbalance, Sunspot zone, Polar field

\section{Introduction}

Magnetic field of the Sun varies with a 22-year periodicity that manifests itself both in the change of signs of leading and following sunspots during solar minimum (the Hale's law), and in the change of sign of the polar field (polar

\footnotetext{
$\triangle$ E.S.Vernova

helena@ev13934.spb.edu

1 IZMIRAN, SPb. Filial, Laboratory of Magnetospheric Disturbances, St. Petersburg, Russian Federation

2 Ioffe Physical-Technical Institute, St. Petersburg, Russian Federation
} 
field reversal) during period of maximum solar activity (see, e.g., Charbonneau, 2010). The distributions of the local and the global magnetic fields exhibit antisymmetry of polarities with respect to the solar equator. At the same time, there exists some asymmetry of the magnetic fields which is reflected in the asymmetric distribution of different forms of solar activity. The north-south asymmetry was discovered in various manifestations of solar activity, such as sunspots, flares, or sudden disappearances of solar prominences (see, e.g., Carbonell et al., 2007; Ballester, Oliver, and Carbonell, 2005; Swinson, Shea, and Humble, 1986; Deng et al., 2016, and references therein).

The magnetic fluxes of the Sun and their imbalance were studied on the basis of different data that characterize the magnetic activity. Asymmetry of the leading and following sunspot polarities in an active region was studied by many authors (see, e.g., van Driel-Gesztelyi and Petrovay, 1990; Fan, 2009).

During the polar field reversals the two hemispheres develop to some extent independently (Svalgaard and Kamide, 2013); as a result the polar fields complete their reversals not synchronously. There exist time intervals when the global solar field loses its dipole structure and behaves like a monopole (Wilcox, 1972; Kotov, 2009). For example, during the solar cycle 24 the south polar field completed its reversal from positive to negative in 2014. In contrast, the north polar field, after a series of reversals changed its sign from negative in 2012 to positive at the beginning of 2015 (Wang, 2017). As emphasized by Kotov (2009), there is no theoretic explanation of the fact that the positive or negative field can dominate practically on the whole Sun for one year or more.

Reversal of the polar magnetic field affects not only the features of solar activity, but also considerably changes the structure of interplanetary space. Significant difference was found in the intensity-time profiles of the galactic cosmic rays around the solar activity minima in the alternate solar magnetic field polarities (Lockwood, Webber, and Debrunner, 2001). One of manifestations of the Sun's magnetic field asymmetry is the displacement of heliospheric current sheet in the south direction (Mursula and Hiltula, 2003; Erdös and Balogh, 2010). Observations of the interplanetary magnetic field (IMF) have suggested a statistical tendency for the heliospheric current sheet to be shifted a few degrees southward of the heliographic equator during the period 1965-2010, particularly in the years near sunspot minimum (Wang and Robbrecht, 2011).

The important information on the asymmetry of the Sun's magnetic field can be obtained by consideration of the magnetic field net flux and its changes in time. Net flux or flux imbalance can be defined as

$$
F_{\text {net }}=\left|F^{\text {pos }}\right|-\left|F^{\text {neg }}\right| .
$$

i.e., as a difference of positive and negative polarity fluxes. The similar parameter was used by Tian, Liu, and Wang (2003) while considering magnetic fluxes for twelve solar active regions in the Cycle 23. It was found that the fluxes of these regions were appreciably imbalanced. For construction of the butterfly diagram (Choudhary, Venkatakrishnan, and Gosain, 2002; Petrie, 2012) signed net flux was used also.

Choudhary, Venkatakrishnan, and Gosain (2002) found, using the magnetograms obtained from the National Solar Observatory at Kitt Peak, that the 
maximum and the median values of the flux imbalance for 137 active regions were respectively $62 \%$ and $9.5 \%$. The $10^{\circ}-40^{\circ}$ active latitudinal zone in the individual hemispheres during the solar maximum showed a flux imbalance of more than $20 \%$. This is reduced to below $10 \%$ when the entire Sun is considered.

When studying hemispheric asymmetry of magnetic fields Petrie and Ettinger (2015) explored correlations between three classes of photospheric magnetic field: active region fields, which are compact, intense, flux-balanced and usually bipolar in structure, located equatorward of about $\pm 30^{\circ}$; polar fields, which are located poleward of $\pm 60^{\circ}$ and are relatively weak, almost unipolar and have large spatial scale, so that over most of the solar cycle they appear dipole-like; and highlatitude surges of field forming from decaying active regions, observed to stream poleward between about $\pm 30^{\circ}$ and $\pm 60^{\circ}$ from active latitudes in plume-like formations.

The present work continues the studies of Vernova, Tyasto, and Baranov (2014) where the problem of polarity imbalance of magnetic flux for the sunspot zone (latitudes form $-40^{\circ}$ to $40^{\circ}$ was considered for the period $1976-2003$ and for fields greater than $100 \mathrm{G}$. Here we used the data of SOLIS which enabled us to continue the studied period till year 2016. Also, somewhat different threshold values were used (fields greater than $50 \mathrm{G}$ and latitudes $5^{\circ}-40^{\circ}$ ). However, the main novelty of the present work is that the polarity imbalance of the sunspot zone is compared with that of the high-latitude fields and their connection with the quadrupole moment is established.

In papers Vernova, Tyasto, and Baranov (2016) and Vernova, Tyasto, and Baranov (2017) the connection of magnetic field intensity with location at certain heliospheric latitudes was studied. It was shown that in the latitudinal distribution of the magnetic field averaged for three cycles strong magnetic fields with strength of $15 \mathrm{G}$ and higher are located in sunspot zone from $5^{\circ}$ to $40^{\circ}$. Strong fields from both sides are surrounded by the weakest fields (less than 5 G: latitudes from $0^{\circ}$ to $5^{\circ}$ and from $40^{\circ}$ to $60^{\circ}$ ). Above $40^{\circ}$ field strength does not exceed $15 \mathrm{G}$, except for a narrow strip of latitudes around $70^{\circ}$ where polar faculae with fields from 15 to $50 \mathrm{G}$ are observed.

In the present paper time-dependencies of the magnetic flux for high-latitude and low-latitude magnetic fields are studied as well as the imbalance of positive and negative fluxes. Following criteria for distinction between low and high latitude regions were used with this aim: low latitudes (sunspot zone) from $5^{\circ}$ to $40^{\circ}$ in each hemisphere; high latitudes from $40^{\circ}$ to $90^{\circ}$ in each hemisphere. The choice of boundary latitude of $40^{\circ}$ allows to consider latitudinal regions with quite different properties of magnetic fields. Only strong fields $(B>50 \mathrm{G})$ were considered in the low latitude region, whereas in the high latitude region the weaker fields $(B<50 \mathrm{G})$ were taken into account. The lower/upper limit of magnetic field intensity $(B=50 \mathrm{G})$ was used to underline a role of strong/weak fields in fluxes of low/high latitudes, accordingly. The choice of this limit makes no major difference and does not influence the main conclusions.

In Section 2 we describe the data and discuss the method applied in the article. Section 3 is devoted to the time changes of absolute values of magnetic fluxes. In Section 4 positive and negative magnetic fluxes and their imbalance for a) high latitudes and b) sunspot zone are considered separately. In Section 5 
we discuss and interpret the obtained results and consider four building blocks of the 22-year magnetic cycle. In Section [6 the main conclusions are drawn.

\section{Data and Method}

For our study we used synoptic maps of the photospheric magnetic field produced at the NSO Kitt Peak (from 1976 to 2016). Data for 1976 - 1977 had many gaps and were not included in our analysis. Combining data sets of two devices, KPVT for 1978 - 2003 (available at ftp://nispdata.nso.edu/kpvt/synoptic/mag/) and SOLIS for 2003 - 2016 (available at https://magmap.nso.edu/solis/archive. html) allowed to study evolution of magnetic fields for nearly four solar cycles. Synoptic maps have the following spatial resolution: $1^{\circ}$ in longitude (360 steps), 180 equal steps in the sine of the latitude from -1 (south pole) to +1 (north pole). Thus, every map consists of $360 \times 180$ pixels of magnetic field strength values in Gauss. On the assumption that the fields are radial to the solar surface, the average line-of-sight component from the original observations was divided by the cosine of the heliocentric angle (the angle between the line-of-sight and the vertical direction).

Noisy values near the poles were detected and replaced by a cubic spline fit to valid values in the polar regions. Although the instrumental noise per resolution element is reduced by the map construction process, the data are less reliable at the poles (in the region with a viewing angle larger than $80^{\circ}$ ) because of noisy measurements associated with the limb and the relatively small number of original measurements that cover the poles.

However, in the sine of the latitude representation of the Kitt Peak data, only one latitude zone corresponds to the range $80^{\circ}-90^{\circ}$, and so this part of the data becomes negligible after the averaging and does not affect the results of the paper. The NSO data are complete in the sense that where observational data are missing, such as when the tilt of the Sun's axis to the ecliptic causes one of the polar regions to be invisible, interpolated values are used (Durrant and Wilson, 2003). Using as initial data magnetic field synoptic maps, we have calculated fluxes for different intensities of magnetic fields and for different latitudinal ranges.

\section{Time changes of magnetic fluxes}

The magnetic field strength for both hemispheres (1978 - 2016) shows a nearly symmetric distribution of positive and negative fields with $65.2 \%$ of the pixels in the strength range $0-5 \mathrm{G}$, whereas pixels with the strength above $50 \mathrm{G}$ occupy only $2.6 \%$ of the solar surface. Magnetic fields in the $5-50 \mathrm{G}$ strength range occupy $32.2 \%$. Strong magnetic fields of both polarities occupy a relatively small part of the Sun's surface. However just the strong magnetic fields of active regions give the main contribution to the total magnetic flux (see Figure11).

Let us consider time changes of the magnetic flux for the whole range of heliolatitudes, but for different values of magnetic field strength. In Figure 1, 


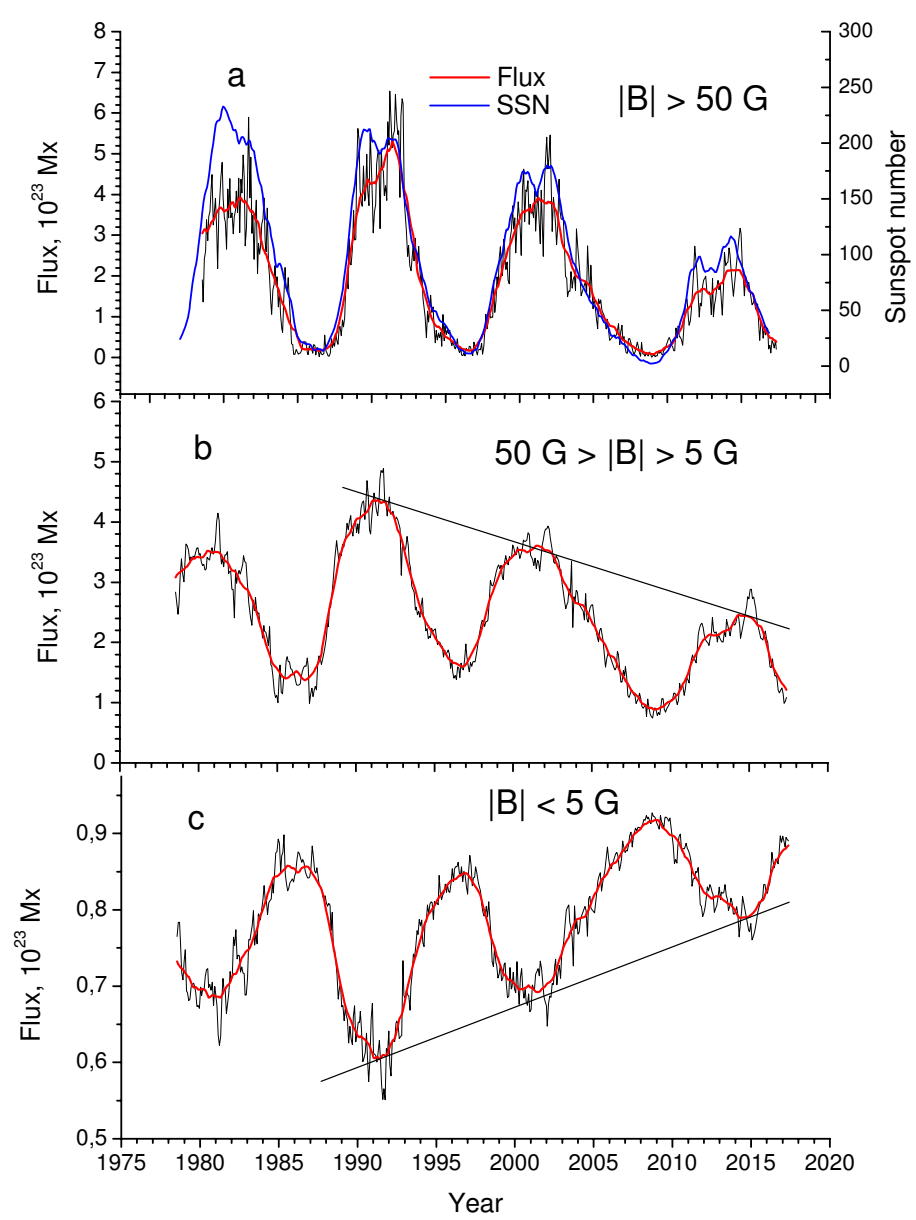

Figure 1. Time changes of the magnetic flux for different ranges of strength (absolute values) for the period 1978 - 2016: (a) fields with $|B|>50 \mathrm{G}$ and sunspot number (SSN); (b) $50 \mathrm{G}>|B|>5 \mathrm{G}$; (c) $|B|<5$ G. Black line - magnetic flux for each Carrington rotation; red line - smoothed curves, which were obtained using 20-rotation running mean.

the flux of the strongest magnetic fields $(B>50 \mathrm{G})$ is presented. Only absolute values of magnetic field strength were considered. For comparison 13-month smoothed monthly sunspot number is shown (http://sidc.oma.be/silso/DATA/ SN_ms_tot_V2.0.txt) by blue line in Figure 10. The magnetic flux tracks the 11year cycle based on sunspot number, reaching a maximum around the time of the second (Gnevyshev) maximum of solar activity (SA). In the minimum of SA the flux falls practically to zero. In three consecutive maxima (Cycles 22, 23 and 24) the flux maximum decreased monotonously more than two times. For the sunspot number this decrease is observed during four solar cycles.

For magnetic fields from 5 to $50 \mathrm{G}$ (Figure1b) time changes closely agree with time changes of the strongest fields (correlation coefficient $R=0.91$ ). Changes with the 11-year cycle and monotonous decrease of the flux maxima can be seen 
for three last solar cycles (noted in Figure 1b by a straight line). The difference between Figures $1 \mathrm{a}$ and $1 \mathrm{~b}$ is that the flux of $5-50 \mathrm{G}$ fields is lower in comparison with the flux of the strongest fields. Other difference is in that the $5-50 \mathrm{G}$ flux does not fall anywhere below a threshold $1 \times 10^{23} \mathrm{Mx}$.

Very special time change display fields with strength $B<5 \mathrm{G}$ which develop in antiphase with the solar cycle (Figure 15). In years when the flux of the strong fields reaches its maximum, fields of $B<5 \mathrm{G}$ have a minimum. The minimum flux increases from Cycle 22 to 24 while for the fields of higher strength the flux maximum falls (compare Figures $1 \mathrm{~b}$ and 15). The maximum values of fluxes of weak magnetic fields $(B<5 \mathrm{G})$ are about ten times lower, than the total flux of stronger fields $(B>5 \mathrm{G})$. Correlation coefficient between fields of $0-5 \mathrm{G}$ and fields $B>50 \mathrm{G}$ is $R=-0.91$, and between fields of $0-5 \mathrm{G}$ and fields of $5-50 \mathrm{G}$ it is equal to $R=-0.98$. Features of the weakest-field change are in accordance with the results obtained by analysis of SOHO/MDI data for 1996 - 2011 which show that magnetic structures with low fluxes change in antiphase with the solar cycle (Jin and Wang, 2014).

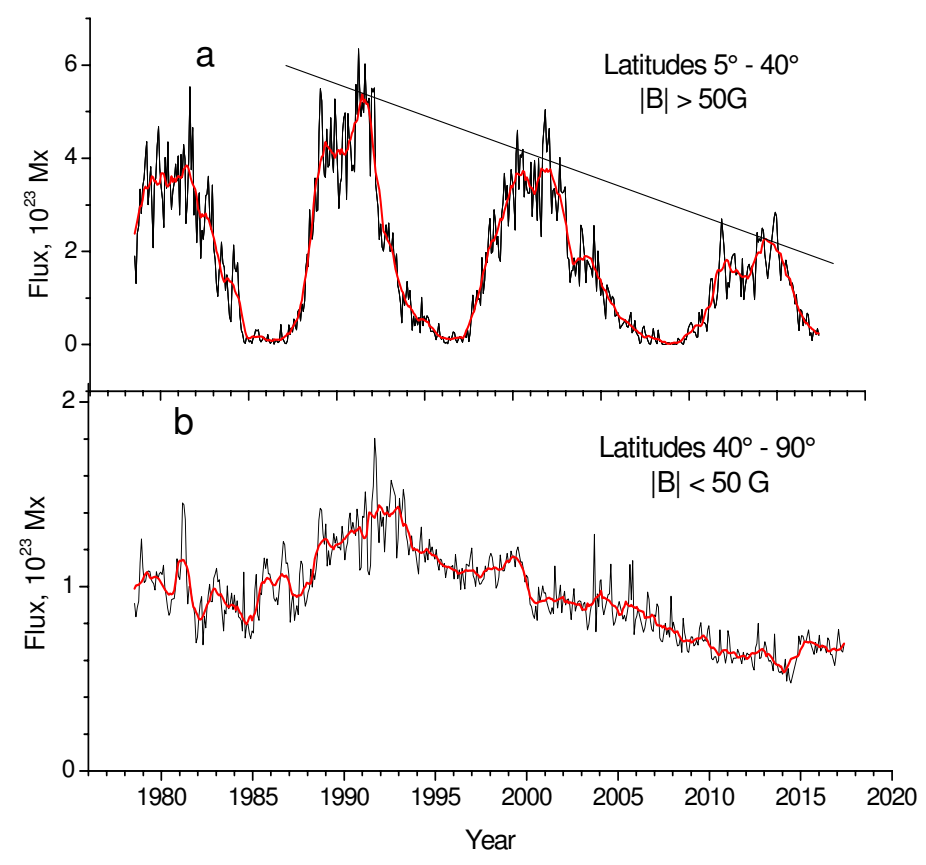

Figure 2. Change of magnetic flux (absolute values) in different latitude ranges for Solar Cycles 21-24: (a) low latitudes $5^{\circ}-40^{\circ}, B>50 \mathrm{G}$; (b) high latitudes $40^{\circ}-90^{\circ}, B<50 \mathrm{G}$. Black line - magnetic flux for each Carrington rotation; red line - 20-point moving average.

In Figure 2 change of magnetic flux for Solar Cycles 21-24 is shown separately for low latitudes $5^{\circ}-40^{\circ}, B>50 \mathrm{G}$ (a) and for high latitudes $40^{\circ}-90^{\circ}, B<50 \mathrm{G}$ (b). Only absolute values of magnetic field strength were considered. For low latitudes the main flux is connected with active regions; thus, the magnetic flux at low latitudes changes with a 11-year cycle (Figure 2a), and the flux maximum 
coincides with the time of the second maximum of Gnevyshev (in the same way as in Figure $1 \mathrm{a}$ ). The time course of magnetic flux for high latitudes (Figure $2 \mathrm{~b}$ ) does not show appreciable recurrence. The basic feature of magnetic flux at high latitudes is monotonous decrease (approximately two times) from a maximum around year 1991, to the minimum values around 2014, that is within 25 years. According to Obridko and Shelting (2009), after 1980 the magnetic moment of the solar dipole showed a tendency to gradually decrease and, in 2007, it has already reached values lower than those at the beginning of the 20th century. The polar field during the Cycle 23 minimum was about $40 \%$ weaker than during two previous cycles (Wang, Robbrecht, and Sheeley, 2009). One can see the general tendencies of magnetic flux decrease both at high, and at low latitudes: falling of the polar field during three cycles coincides with decline of the maxima of these cycles for low latitude fields.

\section{Imbalance of positive and negative magnetic fluxes}

\subsection{High latitudes}

The results obtained previously (Vernova, Tyasto, and Baranov, 2016; Vernova, Tyasto, and Baranov, 2017) show that the latitude $40^{\circ}$ is the boundary, above which the main contribution is made by the magnetic fields lower than $50 \mathrm{G}$.
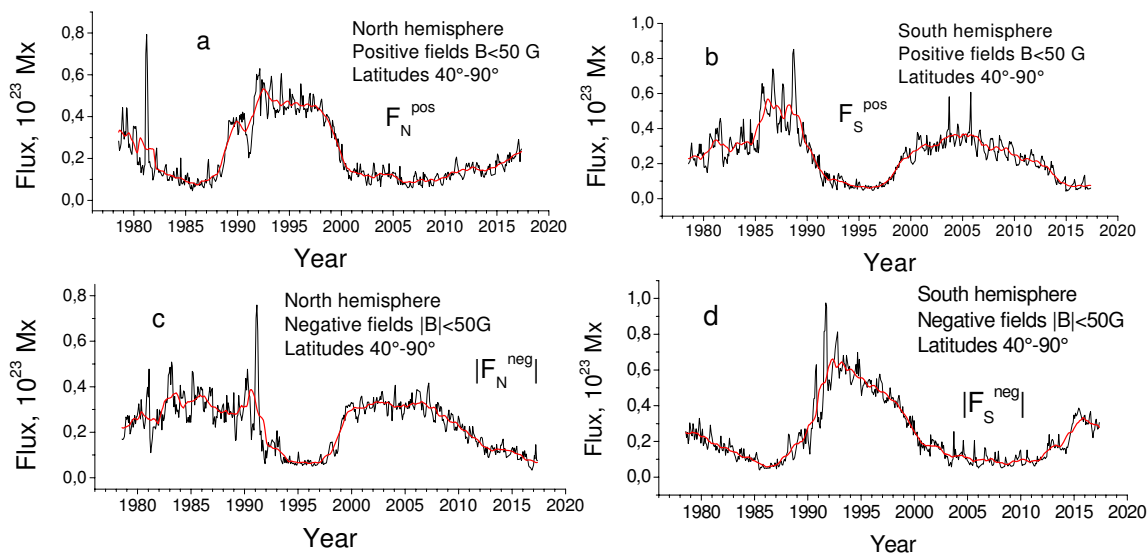

Figure 3. Magnetic fluxes of high-latitude fields from $+40^{\circ}$ to $+90^{\circ}$ and from $-40^{\circ}$ to $-90^{\circ}$ $(B<50 \mathrm{G})$ : (a),(b) - positive fluxes of high latitudes of the northern $\left(F_{N}^{p o s}\right)$ and the southern $\left(F_{S}^{\text {pos }}\right)$ hemispheres; (c), (d) - absolute values of negative fluxes in the northern $\left(\left|F_{N}^{\text {neg }}\right|\right)$ and the southern $\left(\left|F_{S}^{\text {neg }}\right|\right)$ hemispheres. Black lines - flux values for each solar rotation, red lines -20 points smoothing.

At the same time, latitudes from $40^{\circ}$ to $90^{\circ}$ break into three latitudinal strips, each of which corresponds to different intensities of magnetic fields and different manifestations of solar activity: 1) fields of $15-50 \mathrm{G}$ from $65^{\circ}$ to $75^{\circ}$ (polar faculae); 2) fields of $5-15 \mathrm{G}$ from $60^{\circ}$ to $90^{\circ}$ (coronal holes); 3) fields lower 
than $5 \mathrm{G}$ from $40^{\circ}$ to $60^{\circ}$. Time changes and flux imbalance proved to be similar for these field groups which allows to combine the three groups and to study magnetic fluxes of high latitude fields from $+40^{\circ}$ to $+90^{\circ}$ and from $-40^{\circ}$ to $-90^{\circ}(B<50 \mathrm{G})$. In Figures 3 a, b positive fluxes of high latitudes of the northern $\left(F_{N}^{\text {pos }}\right)$ and the southern $\left(F_{S}^{\text {pos }}\right)$ hemispheres are presented. For negative fluxes in Figures 3 , d their absolute values $\left(\left|F_{N}^{n e g}\right|,\left|F_{S}^{n e g}\right|\right)$ are presented.

Positive and negative fluxes for each hemisphere change with the period of 22 years opposite in phases to each other. Transitions from low values to high ones and from high to low values occur quickly, in comparison with wide flat maxima that is most pronounced in the northern hemisphere. It is worth noting the sharp increases of the flux (black line, without smoothing) in the northern hemisphere lasting for several solar rotations in 1981.2 and in 1991.2. The peak in 1981.2 occurred in the positive flux, whereas sharp increase of negative flux produced the peak in 1991.2. Approximately at the same periods of time (in 1982 and 1991) Wang (2004)) found the highest peaks in the IMF strength during last 40 years, and these peaks coincided with peaks in the equatorial dipole strength.

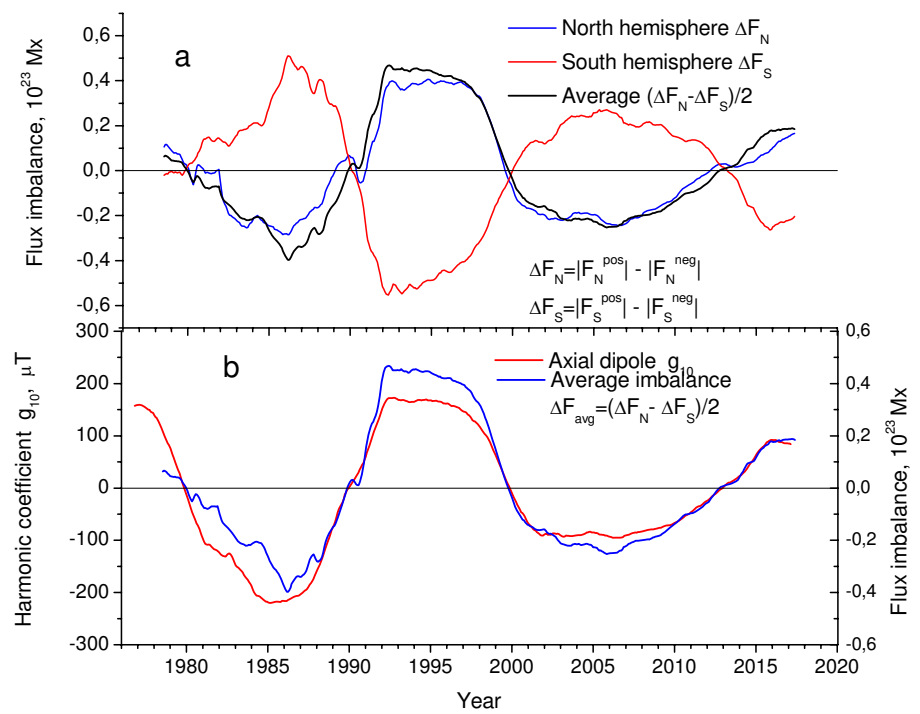

Figure 4. (a) Imbalance of magnetic fluxes for the northern $\left(\Delta F_{N}=F_{N}^{\text {pos }}-\left|F_{N}^{\text {neg }}\right|\right.$, blue curve) and the southern $\left(\Delta F_{S}=F_{S}^{\text {pos }}-\left|F_{S}^{\text {neg }}\right|\right.$, red curve) hemispheres. The average imbalance $\left(\Delta F_{\text {avg }}=\left(\Delta F_{N}-\Delta F_{S}\right) / 2\right.$, black curve). (b) The dipole moment $g_{10}$ of the multipole expansion of the photospheric magnetic field (data of the WSO observatory).

In Figure $4 \mathrm{a}$ the imbalance of magnetic fluxes for the northern $\left(\Delta F_{N}=\right.$ $F_{N}^{\text {pos }}-\left|F_{N}^{\text {neg }}\right|$, blue curve) and the southern $\left(\Delta F_{S}=F_{S}^{\text {pos }}-\left|F_{S}^{n e g}\right|\right.$, red curve) hemispheres are shown. The average imbalance $\left(\Delta F_{\text {avg }}=\left(\Delta F_{N}-\Delta F_{S}\right) / 2\right.$, black curve) changes in phase with the northern hemisphere imbalance. For latitudes above $40^{\circ}$ dominating fields in each hemisphere are those whose sign coincides with the sign of the polar field in this hemisphere. This sign changes once in 11 years after polar field reversal. Thus, the imbalance of positive and negative 


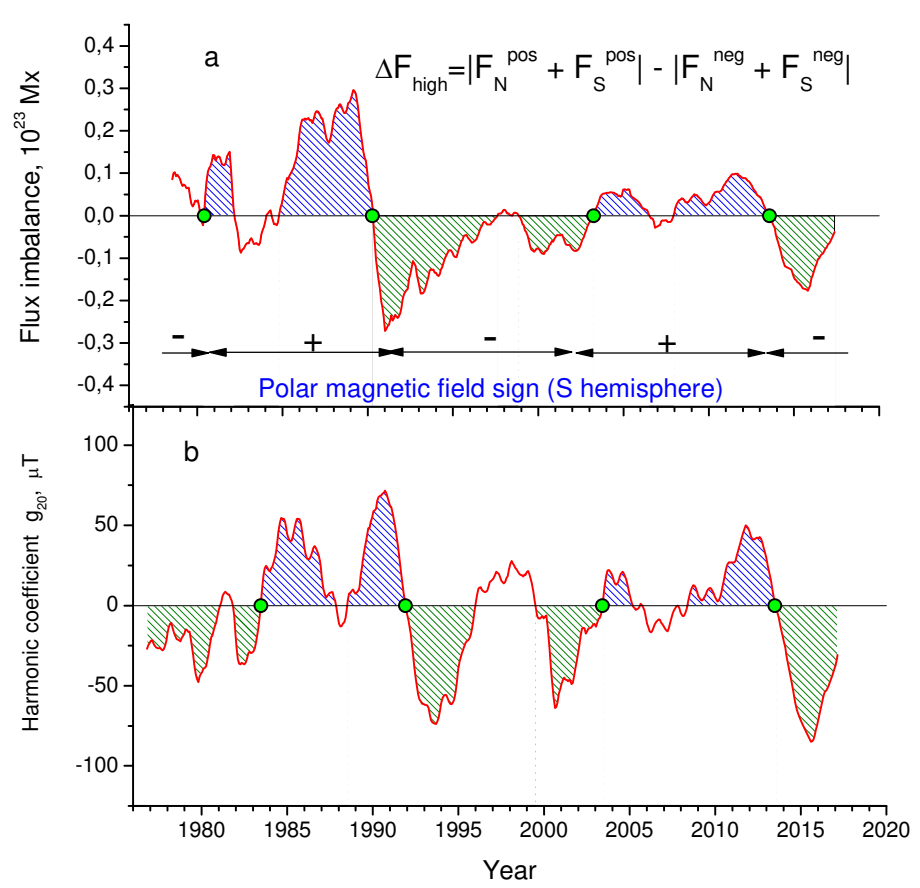

Figure 5. (a) Imbalance $\Delta F_{\text {high }}$ of positive and negative fields of both polar caps for the fields of $B<50 \mathrm{G}\left(\Delta F_{\text {high }}=\left|F_{N}^{\text {pos }}+F_{S}^{\text {pos }}\right|-\left|F_{N}^{\text {neg }}+F_{S}^{\text {neg }}\right|\right)$. (b) Axial quadrupole moment $g_{20}$ of the photospheric magnetic field.

fluxes in a separate hemisphere changes with the 22-year period. The dipole moment $g_{10}$ of the multipole expansion of the photospheric magnetic field according to potential-field source-surface model (PFSS) (Hoeksema and Scherrer, 1986) is presented in Figure 40 (data of the WSO observatory, available at http://wso. stanford.edu/). Comparison of the average imbalance with the dipole moment $g_{10}$ shows their good coincidence (Figure 4b). These results provide evidence that the fields in the latitude range of $40^{\circ}-90^{\circ}$ are directly connected with dipole component of the Sun's magnetic field.

Imbalance $\Delta F_{\text {high }}$ of positive and negative fields of both polar caps for the fields of $B<50 \mathrm{G}$ was calculated (Figure $5 \mathrm{a}$ ) as the sum of the signed high latitude fluxes for the northern hemisphere $F_{N}^{\text {pos }}, F_{N}^{\text {neg }}$ (from $+40^{\circ}$ to $+90^{\circ}$ and for the southern one $F_{S}^{\text {pos }}, F_{S}^{\text {neg }}$ (from $-40^{\circ}$ to $-90^{\circ}$ ):

$$
\Delta F_{\text {high }}=\left|F_{N}^{\text {pos }}+F_{S}^{\text {pos }}\right|-\left|F_{N}^{\text {neg }}+F_{S}^{\text {neg }}\right|
$$

Throughout four solar cycles the strict regularity of the imbalance change is observed (change of the imbalance sign is marked in Figure $5 \mathrm{k}$ by green circles). From one maximum of SA to another the imbalance looks like two peaks of one sign divided by the period of the SA minimum. The sign of the imbalance remains constant during 11 years from one polar field reversal to the other. Thus the full period of change of the imbalance sign makes 22 years. In the lower part 
of Figure $5 \mathrm{k}$, the polarity of the polar magnetic field in the southern hemisphere is displayed. Shading marks time intervals when the imbalance sign coincides with the sign of polar field in the southern hemisphere: positive sign - dark blue shading, and negative sign - green shading. Most of the time the sign of the imbalance coincides with the sign of the polar magnetic field in the southern hemisphere.

In Figure 5b the axial quadrupole moment $g_{20}$ of the photospheric magnetic field is shown (data of the WSO observatory). Good coincidence of the imbalance sign with the sign of the quadrupole moment can be seen. Not only the sign, but also the basic features of the time course of the imbalance and the quadrupole moment are very close. The study of the north-south asymmetry of the solar and heliospheric magnetic field (Bravo and González-Esparza,2000) showed that during activity minima the magnitude of the northern magnetic field is smaller than the magnitude of the southern magnetic field. According to the PFSS model the dominant multipoles are the dipole, the hexapole, and the quadrupole. The sign of the quadrupole component is opposite to that of the dipole and hexapole in the north, but the three components have the same sign in the south which leads to domination of the southern hemisphere fields. This agrees with the fact that the imbalance sign coincides always with the sign of the polar field of the southern hemisphere (Figure 5).

The obtained results allow us to draw the following conclusions for highlatitude fields (from $40^{\circ}$ to $90^{\circ}$ in each hemisphere), $B<50 \mathrm{G}$. The imbalance of positive and negative fields in a separate hemisphere and the total imbalance of high-latitude fields for two hemispheres change with the 22-year period. Change of the sign of the imbalance occurs near reversal of the polar field. However, while in a separate hemisphere the imbalance sign changes similarly to the polar field in this hemisphere and to the dipole moment $g_{10}$, the sign of the total imbalance for two hemispheres always coincides with the sign of the polar field in the southern hemisphere. Change of the sign of the total imbalance coincides with the sign change of the quadrupole moment $g_{20}$.

\subsection{Active zone}

In our papers (Vernova, Tyasto, and Baranov, 2016; Vernova, Tyasto, and Baranov, 2017) it was shown that strong magnetic fields which occupy latitude range from $5^{\circ}$ to $40^{\circ}$ are surrounded from both sides by the weakest fields $(B<5 \mathrm{G}$, latitudes from $0^{\circ}$ to $5^{\circ}$ and from $40^{\circ}$ to $60^{\circ}$ ).

In the present paper for the study of strong fields in the sunspot zone we set the following boundaries: field strength $B>50 \mathrm{G}$ and latitudinal regions from $5^{\circ}$ to $40^{\circ}$. Positive and negative magnetic fluxes of the near-equatorial region $\pm 5^{\circ}$ display special features which we do not consider in this paper.

In Figure 6 fluxes of strong magnetic fields $(B>50 \mathrm{G})$ are presented for the sunspot zone $\left(5^{\circ}-40^{\circ}\right)$. For each synoptic map four different characteristics of magnetic flux were obtained: absolute values of positive and negative fluxes for the northern and the southern hemispheres $-F_{N}^{p o s}, F_{S}^{\text {pos }}, F_{N}^{\text {neg }}, F_{S}^{\text {neg }}$. All these fluxes follow the 11-year cycle of solar activity. Some difference between fluxes can be seen at periods of high solar activity. For each of solar cycles these 


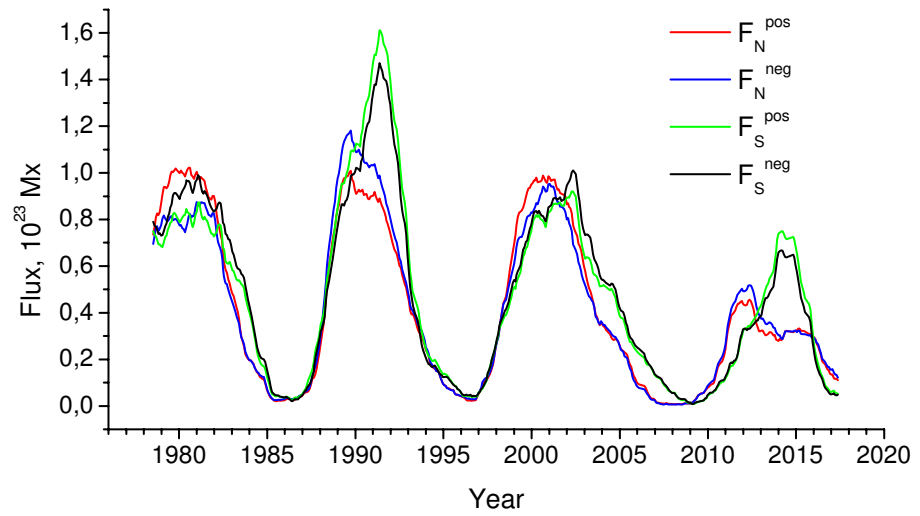

Figure 6. Fluxes of strong magnetic fields $(B>50 \mathrm{G})$ for the sunspot zone $\left(5^{\circ}-40^{\circ}\right)$ : positive $\left(F_{N}^{\text {pos }}, F_{S}^{\text {pos }}\right)$ and negative $\left(F_{N}^{n e g}, F_{S}^{n e g}\right)$ fluxes (absolute values) for the northern and the southern hemispheres.

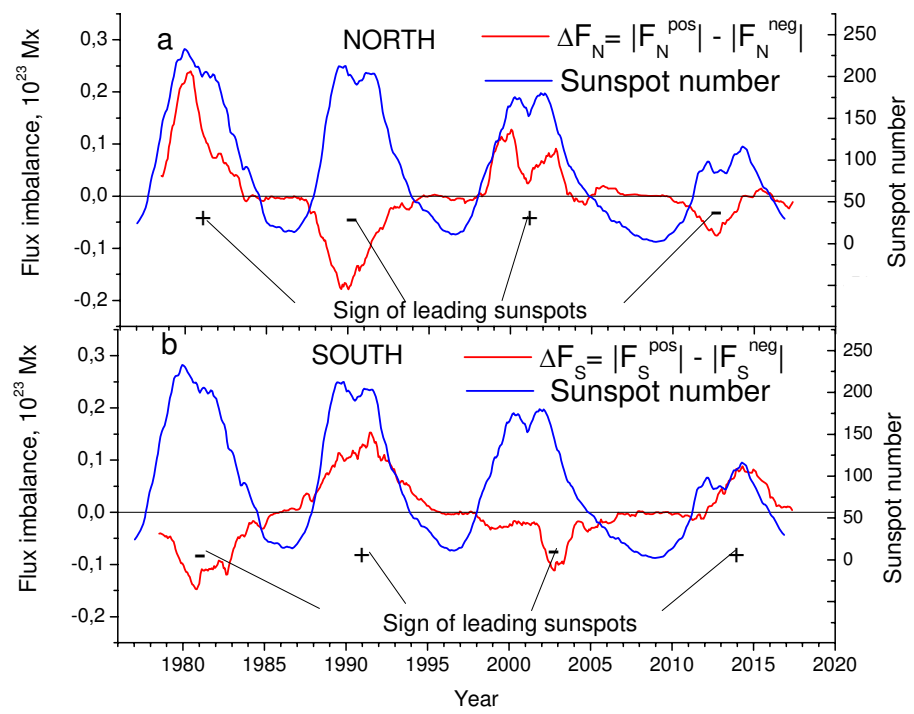

Figure 7. (a) Flux imbalance between positive and negative fluxes for the northern hemisphere $\Delta F_{N}=F_{N}^{\text {pos }}-\left|F_{N}^{n e g}\right|$. (b) Flux imbalance between positive and negative fluxes for the southern hemisphere $\Delta F_{S}=F_{S}^{\text {pos }}-\left|F_{S}^{\text {neg }}\right|$. Blue line denotes sunspot number. The sign of the leading sunspots is shown for each hemisphere.

four fluxes depending on its polarity can be interpreted as the magnetic flux of the leading or the following sunspots of one of the solar hemispheres. The signs of the leading/following sunspots remain constant during solar cycle from one minimum to the next one when sunspots of a new cycle appear with polarities opposite to the previous ones.

Flux imbalance between positive and negative fluxes for the northern hemisphere $\Delta F_{N}=F_{N}^{\text {pos }}-\left|F_{N}^{\text {neg }}\right|$ (Figure $7 \mathrm{a}$ ) varies with the 22-year cycle and 


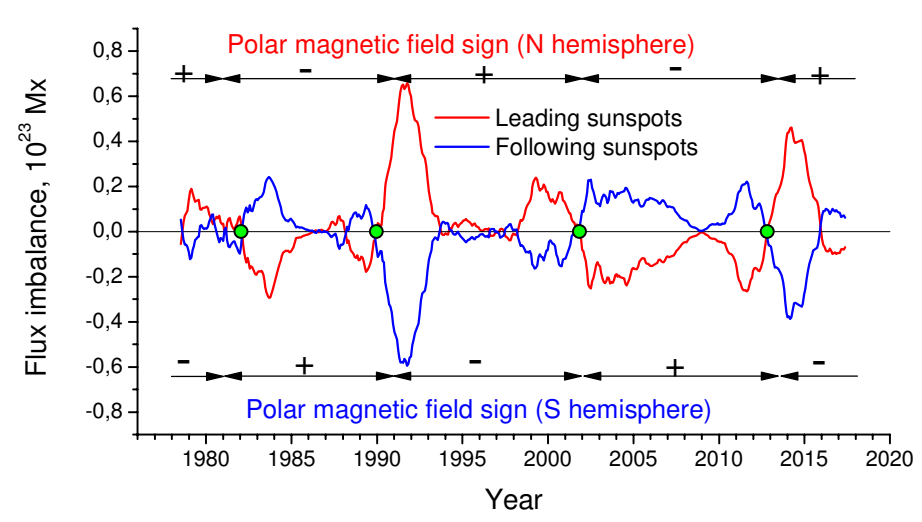

Figure 8. Flux imbalance of leading sunspots of the northern and the southern hemispheres $\Delta F_{\text {lead }}$ (red line) and flux imbalance of following sunspots $\Delta F_{\text {foll }}$ (blue line). Times of polar field reversal are marked by green circles. The signs of the polar magnetic fields in the northern and southern hemispheres are shown, respectively, in the upper part and in the lower part of the figure.

reaches extrema during maxima of solar activity. The flux imbalance passes through zero around the minima of solar activity. Thus, from one minimum to another, the sign of the difference between positive and negative fluxes $\left(\Delta F_{N}\right)$ does not change. In Figure $7 \mathrm{~A}$ the sign of leading sunspots in bipolar sunspot groups is shown. The sign of the flux imbalance coincides with the sign of leading sunspots in the northern hemisphere. Similar results are obtained for the southern hemisphere: the sign of the imbalance $\Delta F_{S}=F_{S}^{\text {pos }}-\left|F_{S}^{\text {neg }}\right|$ always coincides with the sign of leading sunspots (Figure $7 \mathrm{~b}$ ). The imbalances of positive and negative fluxes in each of the solar hemispheres show a 22-year recurrence that is directly connected with the Hale cycle. Evidently, for each of solar hemispheres magnetic flux of the leading sunspots exceeds that of the following sunspots.

There arises a question: if the fluxes of leading sunspots of two hemispheres are compared, which of these fluxes will be higher? It is similarly possible to consider the flux imbalance of following sunspots of two hemispheres. For example, during Solar Cycle 21, leading sunspots of the northern hemisphere had positive polarity (corresponding flux $F_{N}^{\text {pos }}$ ), while the leading sunspots of the southern hemisphere had negative polarity (corresponding flux $F_{S}^{n e g}$ ). Then the imbalance of leading sunspots can be defined as $\Delta F_{\text {lead }}=F_{N}^{\text {pos }}-\left|F_{S}^{\text {neg }}\right|$. During the next solar cycle the leading sunspots in both hemispheres change their polarity. In this case the imbalance of leading sunspots will be $\Delta F_{\text {lead }}=F_{S}^{\text {pos }}-\left|F_{N}^{\text {neg }}\right|$. Flux imbalance of leading sunspots of the northern and the southern hemispheres $\Delta F_{\text {lead }}$ is presented in Figure 8 (red line). Flux imbalance of following sunspots $\Delta F_{\text {foll }}$ (blue line) is in antiphase with the imbalance of the leading sunspot fluxes with correlation coefficient $R=-0.98$. At the time of polar field reversal both parameters change their sign (marked by green circles). The sign of the imbalance between fluxes of leading sunspots of two hemispheres changes with a 22-year magnetic cycle in the same way as the sign of the polar magnetic field in the northern hemisphere (shown in the upper part of Figure 8). The imbalance 
between fluxes of following sunspots repeats the sign of the polar magnetic field in the southern hemisphere (shown in the lower part of Figure 8).

It is possible to show that the imbalance of fluxes of leading sunspots and the imbalance of fluxes of following sunspots define the sign of north-south asymmetry of the magnetic field in the activity zone. North-south asymmetry can be defined as:

$$
\Delta_{N S}=\left(F_{N}^{\text {pos }}+\left|F_{N}^{n e g}\right|\right)-\left(F_{S}^{\text {pos }}+\left|F_{S}^{n e g}\right|\right)
$$

The sign of the imbalance of leading sunspots is opposite to the sign of the imbalance of following sunspots, hence, dominating leading sunspots and dominating following sunspots will have different signs. As fields of leading and following sunspots in the same hemisphere have opposite signs, it follows from this that both fluxes of leading sunspots of a hemisphere, and fluxes of following sunspots of the same hemisphere will dominate simultaneously over corresponding fluxes of the other hemisphere (N-S asymmetry). The similar conclusion was made by Petrie (2012) for magnetic fields during decrease of Cycle 23 and ascent of Cycle 24.

Considering two phases of a 11-year cycle, from a minimum before reversal and from reversal to a minimum, we will show that change of domination of hemispheres (change of N-S asymmetry sign) occurs during polar field reversal and during minimum of SA. Figure 8 shows that the sign of the imbalance of leading sunspots always coincides with the sign of the polar field in the northern hemisphere. It follows that those sunspots which have the same sign, as the polar field of the northern hemisphere will dominate. Thus, from the minimum to the reversal the flux of leading sunspots of the northern hemisphere which has the sign of the polar field in this hemisphere, will always exceed the flux of leading sunspots of the southern hemisphere (the northern hemisphere dominates). After the reversal the sign of the polar field in the northern hemisphere changes and coincides with the sign of leading sunspots in the southern hemisphere (the southern hemisphere dominates from the reversal to the minimum).

During SA minimum the domination returns to the northern hemisphere because leading and following sunspots in each of hemispheres change their signs, whereas the sign of the polar field remains unchanged. As a result north-south asymmetry changes its sign both during polar field reversal and during minimum of SA, so that the northern hemisphere will always dominate from the minimum to the reversal, but the southern hemisphere will dominate from the reversal to the minimum (See Figure 2 in Vernova, Tyasto, and Baranov (2014)).

In the same way as the imbalance of magnetic field for two polar caps $\Delta F_{h i g h}$ (Formula (2)), one can define the total imbalance of positive and negative fields for both sunspot zones $\Delta F_{\text {low }}$ (latitudes from $+5^{\circ}$ to $+40^{\circ}$ and from $-5^{\circ}$ to $\left.-40^{\circ}\right)$ :

$$
\Delta F_{\text {low }}=\left|F_{N}^{\text {pos }}+F_{S}^{\text {pos }}\right|-\left|F_{N}^{\text {neg }}+F_{S}^{\text {neg }}\right|
$$

The total imbalance of two sunspot zones is presented in Figure 9a for magnetic fields $B>50 \mathrm{G}$ (red line). The total imbalance changes in time similarly to the imbalance of leading sunspots, but the imbalance of leading sunspots is about 


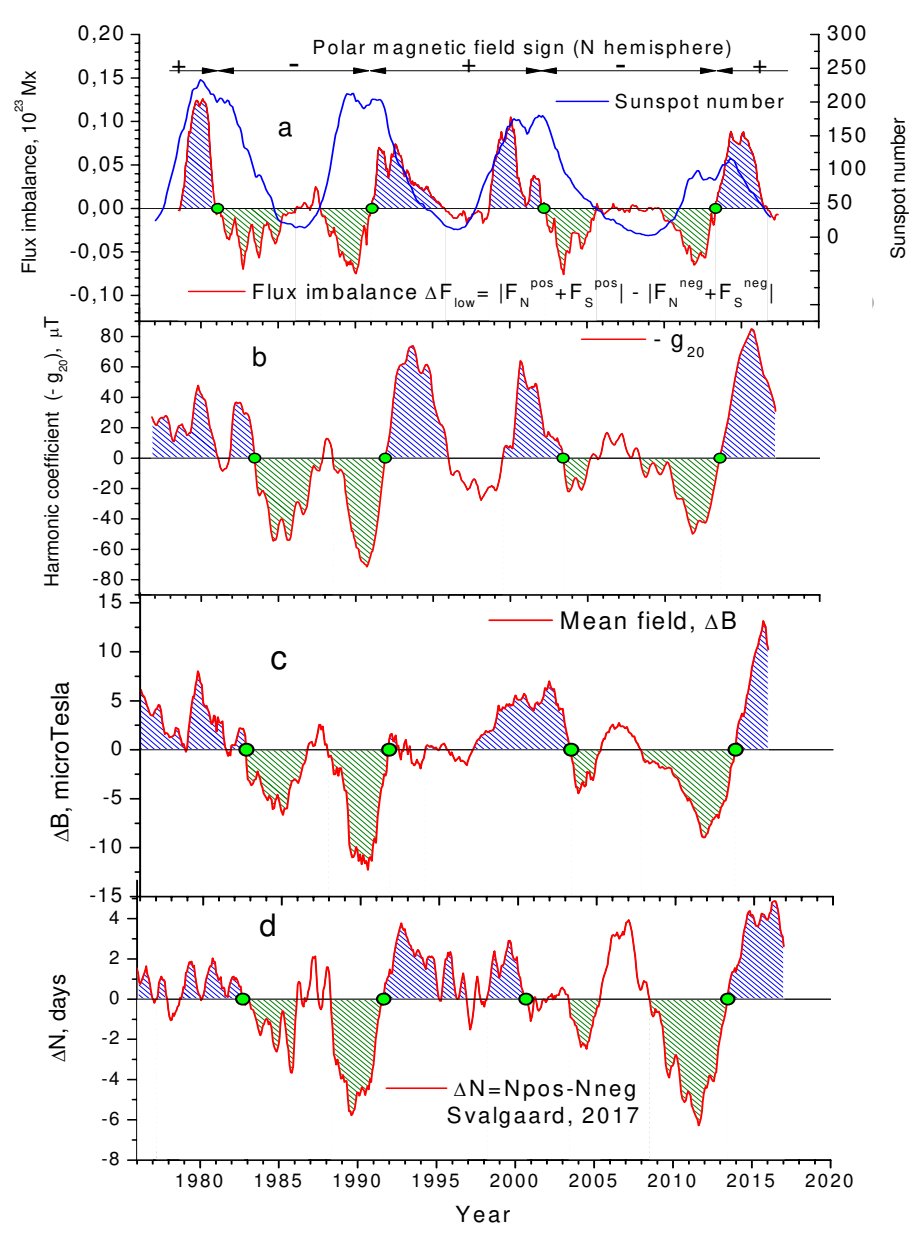

Figure 9. (a) Total imbalance $\Delta F_{\text {low }}=\left|F_{N}^{\text {pos }}+F_{S}^{\text {pos }}\right|-\left|F_{N}^{\text {neg }}+F_{S}^{\text {neg }}\right|$ of two sunspot zones (latitudes from $+5^{\circ}$ to $+40^{\circ}$ and from $-5^{\circ}$ to $-40^{\circ}$ ). Blue line - sunspot number. The sign of the polar field in the northern hemisphere is shown in the upper part of the figure. (b) Reversed quadrupole moment $-g_{20}$. (c) Sun's mean magnetic field (SMMF) $\Delta B$. (d) The difference between numbers of days with positive and negative polarity of the interplanetary magnetic field (according to L. Svalgaard).

five times higher. Change of the imbalance sign (marked in Figure 9 by green circles) occurs during the period close to the polar field reversal. The sign of the polar field in the northern hemisphere is shown in the upper part of Figure $9 \mathrm{k}$. The imbalance sign does not change within 11 years, thus the full period of the sign change makes 22 years. The sign of the imbalance coincides with the sign of the polar field in the northern hemisphere. Time intervals of coincidence are marked by shading for two polarities: positive (blue shading) or negative (green shading). As can be seen from comparison of the flux imbalance with SA cycle (Figure 9a, the dark blue line) the imbalance changes with a strict regularity: each solar cycle contains two parts - one with a positive imbalance, another 
with negative. In Figure $9 \mathrm{~b}$ the change of the quadrupole moment taken with a reversed sign $\left(-g_{20}\right)$ is shown (the shading as in Figure 9a). It is evident that the sign of the magnetic flux imbalance and the sign of the quadrupole moment $\left(-g_{20}\right)$ change with the same periodicity.

To clarify the physical meaning of the imbalance one should compare it with the north-south asymmetry $\Delta_{N S}$. In Figure 8 the change of the imbalances $\Delta F_{\text {lead }}$ and $\Delta F_{\text {foll }}$ is shown. The north-south asymmetry $\Delta_{N S}$ defined by Formula (3) can be rewritten as the difference between $\Delta F_{\text {lead }}$ and $\Delta F_{\text {foll }}$, whereas the total imbalance for sunspot zone (see Figure $9 \mathrm{a}$ ) is given by the sum of $\Delta F_{\text {lead }}$ and $\Delta F_{\text {foll }}$. Since $\Delta F_{\text {lead }}$ and $\Delta F_{\text {foll }}$ have opposite signs (see Figure 8 ), the value of imbalance is significantly lower than that of the north-south asymmetry. Thus, the total imbalance provides a more subtle characteristic of magnetic fields. All the more surprising is that this characteristic has such regular structure and is so closely related with 22-year and 11-year cycles.

It is of interest to compare the flux imbalance with the Sun's mean magnetic field (SMMF). The SMMF represents an imbalance of the magnetic flux $(\Delta B)$ integrated over the entire visible solar disk (Garcia et al., 1999). It is small, less than $\pm 1 \mathrm{G}$, reaching a typical value of about $0.15 \mathrm{G}$ during solar minimum. SMMF is important in many problems of solar physics. Its influence on the interplanetary magnetic field is strong, which can be deduced from a high correlation between the two. Daily values of the mean magnetic field of the Sun (Sun-as-aStar) in microTesla (WSO data: http://wso.stanford.edu/meanfld/MF_timeseries. txt) were averaged over period of one month. For comparison with the imbalance of the sunspot-zone fluxes (Figure $9 \mathrm{a}$ ) the monthly values of $\Delta B$ were smoothed over 20 points (Figure 98). The values of SMMF differ from the threshold of $B>50 \mathrm{G}$ chosen by us; however, the basic features of the imbalance change can be observed in the mean field of the Sun. The sign of the SMMF also displays the 22-year periodicity and changes around the time of the polar field reversal.

The question arises, whether the imbalance of positive and negative fields is a purely local effect or it is reflected in the structure of the heliosphere? To clarify this point, data on the polarity of the IMF (data of L. Svalgaard, see: http:// www.leif.org/research/spolar.txt) were used. The difference between numbers of days with positive and negative polarity of the IMF was calculated (Figure 9d). For convenience of comparison with the flux imbalance (Figure $9 \mathrm{k}$ ) coincidence of polarities is marked by shading. Good agreement between the imbalance and the IMF polarity can be seen, except for years 2005 - 2008 of the prolonged minimum of solar activity. The sign of the difference between positive and negative days of the IMF changes with the 22-year period and coincides mainly with the sign of the polar field in the northern hemisphere. Thus, the imbalance of the magnetic flux of the Sun can be the cause of the asymmetry observed in the IMF.

Results obtained for fields of the sunspot zone (from $5^{\circ}$ to $40^{\circ}$ in each hemisphere), $B>50 \mathrm{G}$ testify:

1) The imbalance in a separate hemisphere changes with the 22-year cycle and the imbalance sign changes near the solar activity minimum. The imbalance sign coincides with the sign of leading sunspots.

2) The sign of the total imbalance for two hemispheres also changes with the 22year cycle, but the sign change occurs near the reversal of the polar magnetic 
field. The imbalance sign always coincides with the sign of the polar field in the northern hemisphere. Total imbalance of positive and negative fluxes shows similar evolution as the quadrupole component $\left(-g_{20}\right)$ of the photospheric magnetic field.

\section{Building blocks of a 22-year cycle}

Different manifestations of the magnetic-flux imbalance considered above, have one common feature: they appear regularly during the period of four solar cycles. We will show that this regularity can be expressed by several simple formulas. Solar dynamo models establish the connection of the 11-year cycle of solar activity to the 22-year cycle of magnetic polarity. The scheme of the polarity change of local and global fields is presented in Figure 10. Two solar cycles are shown in Figure 10k: the even cycle and the following odd cycle. Such choice is connected with the results of Ohl who found a good correlation between SA of two consecutive cycles: an even and the following odd cycle. On the other hand correlation between an odd cycle and the following even one is very weak. According to the Ohl scheme, an even cycle with the succeeding odd cycle form a pair, a single whole (Nagovitsyn, Nagovitsyna, and Makarova, 2009; Ponyavin, 2009). We use number $n$ to denote parity of the cycle. Thus, parity of the solar cycle $n=1$ corresponds to an odd solar cycle, $n=2$ to an even one.

In the course of a 22-year magnetic cycle there are moments when the relation between the polarities of the global and local magnetic fields changes. These moments are related either to the change of the Sun's global magneticfield polarity during high solar activity (polar magnetic-field reversal, marked in Figure 10 by green circles), or to the alternation of the polarities of leading and following sunspots at the solar activity minimum (minima of SA are marked by red circles). In the minimum of SA there is a change of the leading-sunspot sign in each of hemispheres. During the transition from an even to an odd cycle leading sunspots of a new cycle in the northern hemisphere will have the positive sign which coincides with the sign of the polar field in this hemisphere. During the transition from an odd cycle to an even the sign of leading sunspots in the northern hemisphere also will coincide with the sign of the polar field, however it will be a negative sign.

Another situation will take place at the reversal of the polar magnetic field (around maximum of SA). After reversal and up to SA minimum the sign of the polar field in the northern hemisphere will coincide with the sign of leading sunspots in the southern hemisphere. The following is remarkable: in both cases, i.e., throughout all magnetic cycle, leading sunspots of that hemisphere whose sign coincides with the polar-field sign of the northern hemisphere (Figure 10b) will dominate in the magnetic flux. Thus, a 22-year cycle is composed of four intervals (we will call them "building blocks"): during each of these intervals the relation between polarities of the polar magnetic field and of the leading sunspots does not change. These four intervals are from the solar-activity minimum to the magnetic field reversal of the Sun and from the reversal to the next minimum in an even 11-year solar cycle, and analogously for the next odd cycle. The 

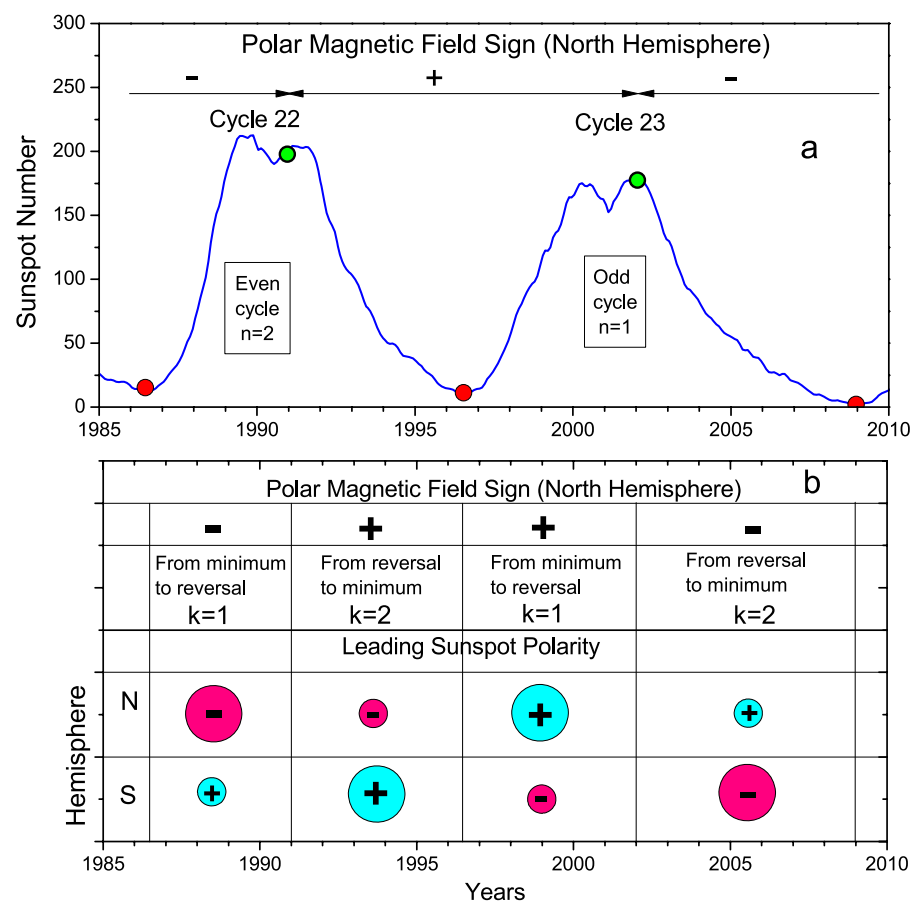

Figure 10. Polar magnetic field sign and leading sunspot polarity for each of the building blocks in Cycles 22 and 23.

relations between the polarities of the global and local magnetic fields will repeat themselves in the next 22-year cycle. Connection of global and local magnetic field polarities in a 22-year magnetic cycle is illustrated by Figure 10p. Each solar cycle can be divided in two parts according to the phase of the cycle: $k=1$ corresponds to the interval of the 11-year cycle from the minimum up to the reversal; $k=2$ to the interval from the reversal up to the minimum. The sign of the polar magnetic field in the northern hemisphere is shown at the top of the Figure 10 and in the scheme of Figure 10b for each of the four quarters of the 22-year magnetic cycle.

The leading sunspots in each of hemispheres are displayed as circles with corresponding polarity. Large circles show dominating leading sunspots. It can be seen that in every building block the sign of the dominating leading sunspots coincides with the sign of the polar magnetic field in the northern hemisphere. The scheme of Figure 10 b shows that at each boundary of two characteristic intervals domination of leading sunspots passes from one hemisphere to the other one. In the first quarter of the 22-cycle the northern hemisphere dominates, in the next quarter the southern hemisphere dominates and so on. As is shown in Figure 8 fluxes of leading and following sunspots in the same hemisphere will dominate simultaneously over corresponding fluxes of leading and following sunspots of the other hemisphere. Thus, from the minimum to the reversal 
the northern hemisphere always dominates, whereas from the reversal to the minimum - the southern hemisphere dominates (N-S asymmetry).

Following simple formulas describe the magnetic field polarities for different phases $(k=1,2)$ of the odd $(n=1)$ and even $(n=2)$ solar cycles:

1. The sign of the polar magnetic field of the northern hemisphere $F_{N}$; the sign of leading-sunspot flux imbalance $\Delta F_{\text {lead }}$; the sign of positive and negative flux imbalance for sunspot zones $\Delta F_{\text {low }}$ (latitudes from $+5^{\circ}$ to $+40^{\circ}$ and from $-5^{\circ}$ to $-40^{\circ}$ ) are determined by two factors: the parity of the solar cycle $n$, and the phase of the 11-year cycle $k$ :

$$
\left.\begin{array}{c}
\operatorname{sign} F_{N} \\
\operatorname{sign} \Delta F_{\text {lead }} \\
\operatorname{sign} \Delta F_{\text {low }}
\end{array}\right\}=(-1)^{n+k} .
$$

2. The sign of the polar magnetic field of the southern hemisphere $F_{S}$; the sign of following-sunspot flux imbalance $\Delta F_{\text {foll }}$; the sign of positive and negative flux imbalance for high latitudes from $+40^{\circ}$ to $+90^{\circ}$ and from $-40^{\circ}$ to $-90^{\circ} \Delta F_{\text {high }}$ are determined by the same two factors $n, k$ :

$$
\left.\begin{array}{c}
\operatorname{sign} F_{S} \\
\operatorname{sign} \Delta F_{\text {foll }} \\
\operatorname{sign} \Delta F_{\text {high }}
\end{array}\right\}=(-1)^{n+k+1} .
$$

3. The sign of leading sunspots in the northern hemisphere; the sign of imbalance between positive and negative fluxes in the northern hemisphere $\Delta F_{N}$ are determined by the parity of the solar cycle $n$ :

$$
\left.\begin{array}{c}
\operatorname{sign}(\text { lead. sunspot }) \\
\operatorname{sign} \Delta F_{N}
\end{array}\right\}=(-1)^{n+1}
$$

4. The sign of leading sunspots in the southern hemisphere; the sign of imbalance between positive and negative fluxes in the southern hemisphere $\Delta F_{S}$ are determined by the parity of the solar cycle $\mathrm{n}$ :

$$
\left.\begin{array}{l}
\operatorname{sign}(\text { lead. sunspot }) \\
\operatorname{sign} \Delta F_{S}
\end{array}\right\}=(-1)^{n} .
$$

5. The sign of the north-south asymmetry depends on the phase of the 11-year cycle $\mathrm{k}$ (before or after the reversal):

$$
\operatorname{sign} \Delta_{N S}=(-1)^{k+1}
$$

6. Previously we considered the longitudinal distribution of sunspots (Vernova et al., 2004) and of photospheric magnetic fields (Vernova, Tyasto, and Baranov, 2007). The longitudinal distribution for the ascending phase and the maximum sharply differ from the longitudinal distribution for the descending phase and the minimum of the solar cycle. Active longitudes change by $180^{\circ}$ when SA evolves from the ascending phase to the descending one. The maximum of the longitudinal distribution is attained at $180^{\circ}$ for the ascending phase and the 
maximum of the solar cycle when the polarities of the leading sunspot and of the global field coincide in each hemisphere, and at $0^{\circ} / 360^{\circ}$ for the descending phase and the minimum when these polarities are opposite. The active longitude is determined by the phase of solar cycle $k$ :

$$
\text { Active Longitude }=\pi k
$$

The above formulas evidence that four building blocks of the 22-year magnetic cycle manifest itself in periodic changes of magnetic field polarities. We believe that representation of the 22-year magnetic cycle as consisting of four characteristic intervals (building blocks) can be useful for studying the processes underlying the observed changes of magnetic field polarities. This representation does not merely establish the fact of alternation of global and local field polarities, but also states the change of domination of certain magnetic field group that is reflected in changes of solar activity and solar-terrestrial relations. In fact, many observations show that behavior of the solar activity manifestations changes significantly both for different phases of the solar cycle and for the odd and even cycles.

Some examples of this connection are presented below. Difference between phases of ascent and descent leads to occurrence of a hysteresis for many indexes of SA (Bachmann et al.,2004). The hysteresis effect that shows up as a nonunique relationship among the emissions from the photosphere, chromosphere, and corona during the rising and declining phases of solar and stellar activity was analyzed by Bruevich and Yakunina (2016). Solar-terrestrial relations also display the dependence on the phase of the cycle. It was shown that ionospheric indices fo2 and Ap weakly depend on the level of solar activity, but the effect of hysteresis is clearly seen (Bruevich et al., 2016). The number of M-class and $\mathrm{X}$-class flares tends to follow the sunspot number, but there is a tendency to have more flares on the declining phase of a sunspot cycle (Hathaway, 2015).

The 22-year periodicity of SA displays itself as the difference between two successive maxima of the 11-year cycle, which follow the Gnevyshev-Ohl rule: the maximum of an even cycle is lower than the maximum of the following odd cycle. Studying the Fraunhofer lines in the solar spectrum variations Livingston et al. (2007) found that solar minimum around 1985 was clearly seen in the high photosphere lines, but the following minimum in 1996 was missing, perhaps indicating a role for the 22-year Hale cycle.

Cosmic-ray modulation shows strong dependence both on the 11-year cycle of SA, and on the 22-year magnetic cycle. When the solar magnetic field in the northern hemisphere is positive, the galactic cosmic rays drift downward from the solar poles toward the Earth and then out along the warped current sheet to the boundary of the heliosphere (Lockwood, Webber, and Debrunner, 2001). When it is negative, the drift pattern is the opposite. The difference in the appearance of the two halves of the 22-year solar magnetic cycle, peaked and flat-topped, supports the role of drifts near the cosmic ray intensity maxima. The intensity of cosmic rays at the solar activity minima also depends on the solar magnetic field polarity. Singh, Badruddin, and Ananth (2005) observed differences between time lags of the solar activity and cosmic ray intensity in 
odd and even cycles as well as differences in the shape, size etc. of hysteresis loops during odd and even cycles. The time lag between cosmic ray intensity and the solar index is different in odd (10 - 14 months) and even ( $1-4$ months) cycles. Differences in time lag between periods of $A<0$ polarity ( $9-10$ months) and $A>0$ polarity ( $3-5$ months) was also found $(A>0$ corresponds to the positive polarity of the polar field in the north hemisphere). These examples show the influence of different phases of the 22-year magnetic cycle on solar activity and heliosphere.

\section{Conclusions}

In this paper we studied polarity imbalance of photospheric magnetic field for high latitudes (from $40^{\circ}$ to $90^{\circ}$ in each hemisphere) and for sunspot zone (from $5^{\circ}$ to $40^{\circ}$ in each hemisphere) during four solar cycles (Solar Cycles 21-24). We used the threshold $B<50 \mathrm{G}$ for high latitudes and $B>50 \mathrm{G}$ for the sunspot zone. For these two latitude zones we considered imbalance of positive and negative magnetic fields for each hemisphere and for both hemispheres together. For sunspot zone we calculated polarity imbalance for leading sunspots of two hemispheres as well as for following sunspots. All these polarity imbalances display regular structure and change with the 22-year period.

The threshold $40^{\circ}$ separates two latitude intervals whose imbalances $\left(\Delta F_{\text {high }}\right.$ and $\left.\Delta F_{\text {low }}\right)$ develop in antiphase. The sign of the total imbalance for high latitudes (from $40^{\circ}$ to $90^{\circ}$ and from $-90^{\circ}$ to $-40^{\circ}$ ) coincides with the sign of the polar field in the southern hemisphere and also with the imbalance of following sunspots according to Formula (6). On the other hand, the sign of the imbalance for sunspot zone (from $5^{\circ}$ to $40^{\circ}$ and from $-40^{\circ}$ to $-5^{\circ}$ ) coincides with the sign of the polar field in the northern hemisphere as well as with the imbalance of leading sunspots (see Formula (50). We obtained a good agreement of the imbalance of high-latitude fields with the quadrupole moment $g_{20}$, while for low-latitude fields there is an agreement with $-g_{20}$, the quadrupole moment taken with the opposite sign.

It was noted in Virtanen and Mursula (2016) and Wang (2014) that the quadrupole component plays in important role in the shift of the heliospheric current sheet southward. Our paper reveals a very close relation of the magneticfield imbalance with the quadrupole moment. It looks reasonable to suggest that just the imbalance of sunspot-zone fields, which always shows domination of the fields with the same sign as the polar field in the northern hemisphere, results in the southward shift of the heliospheric current sheet.

Imbalances for high latitudes and for sunspot zone have opposite signs and, as a result, the imbalance for all latitudes has lower values. However, while magnetic flux for sunspot zone is approximately three times greater than magnetic flux for high latitudes (Figure2), the total imbalance for sunspot zone is about two times less than the total imbalance for high latitudes (see Figure 5a and Figure 97). This difference between the imbalances for sunspot zone and for high latitudes (which is especially clearly seen in Cycle 22) shows that total imbalance for the whole range of latitudes cannot be zero. 
In the course of a 22-year magnetic cycle there are four intervals (from the solar-activity minimum to the magnetic-field reversal of the Sun and from the reversal to the next minimum in an even 11-year solar cycle, and analogously for the next odd cycle) when the relation between polarities of the polar magnetic field and of the leading sunspots does not change. We believe that this representation of the 22-year magnetic cycle as consisting of the four characteristic intervals ("building blocks") can be useful in the study of the processes on the Sun and of solar-terrestrial relations.

The obtained results show that in addition to well-known 22-year periodic patterns in the change of global and local fields there also exists a 22-year periodicity in the change of imbalance of both high-latitude and low-latitude fields.

Acknowledgments NSO/Kitt Peak data used here are produced cooperatively by NSF/NOAO, NASA/GSFC, and NOAA/SEL. This work utilizes SOLIS data obtained by the NSO Integrated Synoptic Program (NISP), managed by the National Solar Observatory, which is operated by the Association of Universities for Research in Astronomy (AURA), Inc. under a cooperative agreement with the National Science Foundation. Wilcox Solar Observatory data used in this study was obtained via the web site http://wso.stanford.edu courtesy of J.T. Hoeksema. We are thankful to Prof. L. Svalgard for providing data on the IMF sector structure polarity.

\section{References}

Bachmann, K.T., Maymani, H., Nautiyal, K., te Velde, V.: 2004, Adv. Space Res. 34, 274. ADS DOI

Ballester, J.L., Oliver, R., Carbonell, M.: 2005, Astron. Astrophys. 431, L5-L8. ADS, DOI

Bravo, S., González-Esparza, J.A.: 2000, Geophys. Res. Lett. 27, 847. ADS, DOI.

Bruevich, E.A., Yakunina, G.V.: 2016, Astrophysics 59, 369. ADS DOI

Bruevich, E.A., Kazachevskaya, T.V., Katyushina, V.V., Nusinov, A.A., Yakunina, G.V.: 2016, Geomagnetism Aeronomy 56, 1075. ADS DOI

Carbonell, M., Terradas, J., Oliver, R., Ballester, J.L.: 2007, Astron. Astrophys. 476, 951. ADS DOI

Charbonneau, P.: 2010, Living Rev. Solar Phys. 7, 3. ADS, DOI

Choudhary, D.P., Venkatakrishnan, P., Gosain, S.: 2002, Astrophys. J. 573, 851. ADS, DOI.

Deng, L.H., Xiang, Y.Y., Qu, Z.N., An, J.M.: 2016, Astron. J. 151, 70. ADS, DOI

Durrant, C.J., Wilson, P.R.: 2003, Solar Phys. 214, 23. ADS DOI

Erdös, G., Balogh,A.: 2010, J. Geophys. Res. 115, A01105. ADS, DOI

Fan, Y.: 2009, Living Rev. Solar Phys. 6, 4. ADS, DOI

Garcia, R.A., Boumier, P., Charra, J., Foglizzo, T., Gabriel, A.H., Grec et al.: 1999, Astron. Astrophys. 346, 626. ADS,

Hathaway, D.H.: 2015, Living Rev. Solar Phys., 12, 4. ADS DOI

Hoeksema, J.T., Scherrer, P.H.: 1986, Solar Magnetic Field - 1976 through 1985. Report UAG-94, WDCA, Boulder, USA. ADS.

Jin, C.L., Wang, J.X.: 2014, J. Geophys. Res.: Sp. Phys. 119, 11. ADS, DOI

Kotov, V.A.: 2009, Bull. Crimean Astrophys. Observatory 105, 45. ADS DOI

Livingston, W.; Wallace, L.; White, O. R.; Giampapa, M.S.: 2007, Astrophys. J. 657, 1137. ADS DOI

Lockwood, J.A., Webber, W.R., Debrunner, H.: 2001, J. Geophys. Res. 106, Iss. A6, 10635. ADS DOI

Mursula, K., Hiltula, T.: 2003, Geophys. Res. Lett. 30(22), SSC 2-1. ADS, DOI

Nagovitsyn, Yu.A., Nagovitsyna, E.Yu., Makarova, V.V.: 2009, Astronomy Letters 35, 564. ADS DOI 
Obridko, V.N., Shelting, B.D.: 2009, Astronomy Letters 35, Iss. 4, 247. ADS DOI

Petrie, G.J.D.: 2012, Solar Phys. 289, 577. ADS, DOI

Petrie, G., Ettinger, S.: 2015, Space Sci. Rev. 210, 77. ADS DOI

Ponyavin, D.I.: 2009, Adv. Space Res. 29, 421. ADS DOI

Singh, M.; Badruddin; Ananth, A.G.: 2005, Proceedings of the 29th International Cosmic Ray Conference. Pune, India. Volume 2, 139. ADS

Svalgaard, L., Kamide, Y.: 2013, Astrophys. J. 763, 23. ADS, DOI.

Swinson, D.B., Shea, M.A., Humble, J.E.: 1986, J. Geophys. Res. 91, 2943. ADS DOI.

Tian, L., Liu, Y., Wang, H. : 2003, Solar Phys. 215, Iss. 2, 281. ADS DOI

van Driel-Gesztelyi, L., Petrovay, K.: 1990, Solar Phys. 126, 285. ADS. DOI

Vernova, E.S., Mursula, K., Tyasto, M.I., Baranov, D.G.: 2004, Solar Phys. 221, 151. ADS DOI

Vernova, E.S., Tyasto, M.I., Baranov, D.G.: 2007, Solar Phys. 245, 177. ADS. DOI

Vernova, E.S., Tyasto, M.I., Baranov, D.G.: 2014, Solar Phys. 289, 2845. ADS DOI

Vernova, E.S., Tyasto, M.I., Baranov, D.G.: 2016, Solar Phys. 291, 741. ADS DOI

Vernova, E.S., Tyasto, M.I., Baranov, D.G.: 2017, arXiv:1704.02586.

Virtanen, I., Mursula, K.: 2016, Astron. Astrophys. 591, id.A78. ADS DOI

Wang, Y.-M.: 2004, Solar Phys. 224, 21. ADS, DOI

Wang, Y.-M.: 2014, Space Sci Rev. 186, 387. ADS DOI

Wang, Y.-M.: 2017, Space Sci. Rev. 210, 351. ADS, DOI

Wang, Y.M., Robbrecht, E.: 2011, Astrophys. J. 736, 136. ADS, DOI

Wang, Y.-M., Robbrecht, E., Sheeley, N.R., Jr.: 2009, Astrophys. J. 707, 1372. ADS, DOI

Wilcox, J.M.: 1972, Comments on Astrophysics and Space Physics 4, 141. ADS. 